\title{
Lasing in a hybrid-aligned cholesteric
}

\author{
${ }^{1}$ Nastishin Yu. A., ${ }^{2}$ Dudok T. H., ${ }^{1}$ Hrabchak V. I., ${ }^{3}$ Lychkovskyy E., \\ ${ }^{1}$ Yakovlev M. Yu., ${ }^{1}$ Vankevych P. I., ${ }^{4}$ Meyer C. and ${ }^{5}$ Pansu B. \\ ${ }^{1}$ Hetman Petro Sahaidachnyi National Army Academy, 32 Heroes of Maidan Street, \\ 79012 Lviv, Ukraine \\ ${ }^{2}$ Vlokh Institute of Physical Optics, 23 Dragomanov Street, 79005 Lviv, Ukraine \\ ${ }^{3}$ Lviv Danylo Halytsky National Medical University, 69 Pekarska Street, 79010 \\ Lviv, Ukraine \\ ${ }^{4}$ Laboratoire de Physique des Systemes Complexes, Universite de Picardie, Jules \\ Verne, 33 rue Saint-Leu, 80039 Amiens, France \\ ${ }^{5}$ Laboratoire de Physique de Solides, CNRS, Univ. Paris-Sud, Université Paris- \\ Saclay, Orsay F-91405, France
}

Received: 26.05 .2017

\begin{abstract}
Optically pumped laser generation at a long-wavelength edge of cholesteric photonic bandgap is detected in a dye-doped cholesteric cell with a hybrid alignment, where long axes of cholesteric molecules are parallel to one of the substrates and perpendicular to the opposite substrate in its close vicinity. The hybrid director alignment in the cell is confirmed by polarization optical microscopy observations in both reflection and transmission modes.
\end{abstract}

Key words: lasing in liquid crystals, photonic bandgap, polarization optical microscopy, liquid crystal textures, hybrid director alignment

PACS: $42.55 . \mathrm{Zz}$

UDC: $538.958+681.7 .069 .24$

\section{Introduction}

Lasing in dye-doped cholesteric $(\mathrm{Ch})$ liquid crystals is usually obtained in cells with uniform planar (i.e., parallel to substrates) Ch layers. Due to the phenomenon of selective light reflection and under the conditions of suitable relationships between the $\mathrm{Ch}$ pitch and the light wavelength, such planar Ch layers form a resonator for circularly polarized light waves of the same handedness as that of the Ch chirality [1-4]. Quality of the planar alignment is therefore one of the key factors for lasing [5] in cholesterics (Ch-s). Perfect enough planar alignment is hard to achieve for thick cells. For this reason, relatively thin cells with the thicknesses $\sim 5-15 \mu \mathrm{m}$ are usually used for lasing.

Recently we have shown $[6,7]$ that laser generation can also be obtained in thick imperfectly aligned cells with a large number of defects. In this study, we check whether this effect is possible in a dye-doped $\mathrm{Ch}$ cell with a hybrid surface alignment. In the latter case, the long axes of $\mathrm{Ch}$ molecules are parallel to one of the substrates but perpendicular near the opposite substrate, thus revealing a homeotropic alignment here. Then the $\mathrm{Ch}$ layers are expected to be strongly curved along the cell-thickness direction. Up to date, there is no evidence that the cell with the substrates providing the hybrid alignment for nematics does the same effect for short-pitch Ch-s. Moreover, the short-pitch Ch-s show specific elasticity and surface-anchoring properties if compared with their long-pitch and untwisted (namatic) counterparts [8].

Identification of the hybrid alignment in itself represents another complicated issue for nematics [9]. The problem is that the polarization optical microscopy (POM) textures of the hybrid 
nematic cells are qualitatively not distinguishable from those of the cells with uniformly tilted or even planar director. Special techniques such as flourescent polarization confocal microscopy [10, 11], PolScope $[12,13]$ or magnetic null technique $[9,14-16]$ have to be applied to detect hybridity in a nematic cell. For Ch-s, especially those with short pitches, it still remains an open problem. Here we will demonstrate that the hybrid-aligned short-pitch Ch cells show distinctive POM textures in both the transmission and reflection modes, which can allow for unambiguous identification of their hybrid alignment.

At first glance, the $\mathrm{Ch}$ cell with the hybrid alignment should not reveal lasing, because distortion of $\mathrm{Ch}$ layers along the cell normal could violate standard demands for a resonator. Surprisingly, we have still detected clear lasing peaks for the hybrid cell at the edge of its $\mathrm{Ch}$ photonic bandgap (PhBG), similar to those observed for the planar $\mathrm{Ch}$ cells. The POM textures and the experimental registration of the lasing effect in the hybrid Ch cells reported in this study suggest that the layers remain planar for some finite micron distance from substrate near the substrate with planar alignment.

\section{Experimental}

We prepared a cell assembled of two substrates. One of them was coated with a polymer SE5661 and provided homeotropic orientation of molecules at the surface, whereas the other was coated with a polymer PI2555 and aligned nematic molecules parallel to its surface. The gap between the substrates was fixed with spherical glass spacers of calibrated diameter, which were added into a UV-sensitive glue applied between the substrates along their edges. After photo-polymerization of the glue with a UV-lamp, the cell thickness was calculated to be $d=16.6 \mu \mathrm{m}$, using a light interference technique and spectral transmission data.

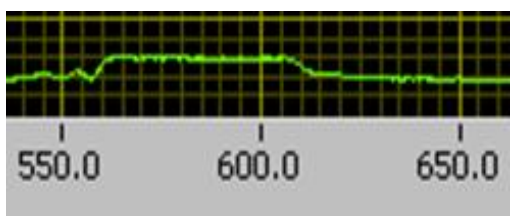

(a)

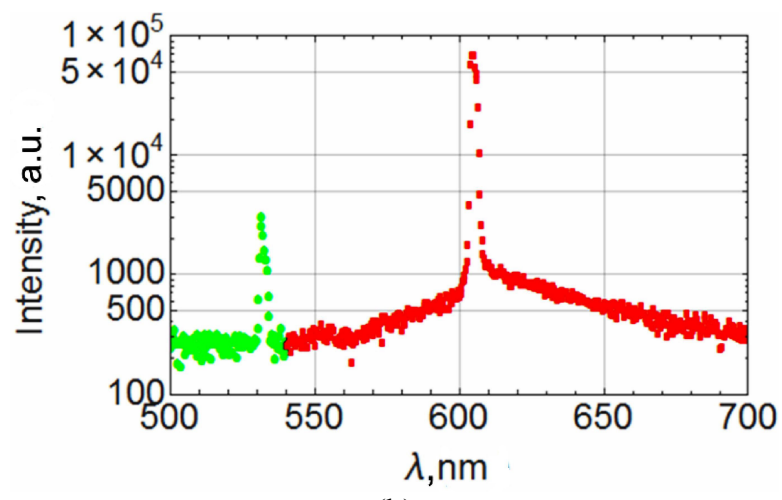

(b)

Fig. 1. (a) Reflection spectrum of a planar-oriented Ch cell filled with a mixture HNG751600-100 + 24\% S811. The abscissa axis corresponds to light wavelength in nanometres and the ordinate to reflected light intensity in arbitrary units; (b) Emission spectra of a planar Ch cell (HNG751600-100 + 24\% S811 + 0.5\% $\mathrm{NR}$ ). A lasing line is observed at $\lambda=605 \mathrm{~nm}$ (red dots), i.e. at a long-wavelength edge of Ch PhBG. A peak at $\lambda=532 \mathrm{~nm}$ (green dots) corresponds to the light of excitation laser.

The gap of the cell was filled with a HNG751600-100 nematic mixed with 24 wt. \% of a chiral agent S811 and 0.5 wt. \% of a fluorescent dye Nile Red. The spectral band of selective reflection for the $\mathrm{Ch}$ with no dye in the planar cell was measured using a fibre micro-spectrometer Ocean Optics USB 2000. The band is located between 560 and $605 \mathrm{~nm}$ (see Fig. 1a). For the Ch-s, which represent $1 \mathrm{D}$ photonic crystals, the location of the selective reflection band visualizes the position of the $\mathrm{Ch} \mathrm{PhBG}$. 
The composition of the $\mathrm{Ch}$ mixture was chosen such that the maximum of the fluorescence emission band for the NR dye in the liquid-crystal matrix corresponded to a long-wavelength edge of the Ch PhBG. Under excitation with green $(\lambda=532 \mathrm{~nm})$ light, the fluorescence emission band maximum for the NR dye dispersed in the liquid-crystal matrix was located at $610 \mathrm{~nm}$. As expected, laser generation in the planar $\mathrm{Ch}$ cell with the same composition (HNG751600$100+24 \% \mathrm{~S} 811+0.5 \% \mathrm{NR}$ ) was detected at the long-wavelength edge of the Ch PhBG (see Fig. 1b). We used for pumping a pulse laser with the wavelength $\lambda=532 \mathrm{~nm}$, the pulse width $1 \mathrm{~ns}$, the tunable $(1 \mathrm{~Hz} \div 20 \mathrm{kHz})$ repetition frequency and the pulse energy up to $2 \mu \mathrm{J} /$ pulse. The repetition frequency was chosen to be $150 \mathrm{~Hz}$.

\section{Results and discussion}

\subsection{Identification of hybrid alignment}

As seen from Fig. 2, the POM observations in the transmission mode reveal that the texture of the cell resembles a typical $\mathrm{Ch}$ texture, indicating planar $\mathrm{Ch}$ layers (with helical axis perpendicular to the substrates) and a so-called 'Grandjean texture' with clearly visible 'oily streaks' (see thick lines in Fig. 2) against the background of several thin lines. This resembles a 'fingerprint' texture, i.e. a typical texture indicating homeotropic $\mathrm{Ch}$ layers (with helical axis parallel to the substrates). For the reasons explained below, we refer to it as to a 'pseudo-fingerprint' texture. Moving the focus of the microscope up and down, one can see that these two textures are located at different levels in the cell. It looks like the Grandjean texture is located near the substrate that requires the planar orientation of molecules on its surface, whereas the 'pseudo-fingerprint texture' is formed near the substrate with the hometropic-anchoring conditions. A homogeneous purple colour of the texture in Fig. 2 is a result of selective reflection. A combination of these two textures (the Grandjean texture against the background of the 'pseudo-fingerprint' texture) is an indirect evidence of the hybrid surface alignment.

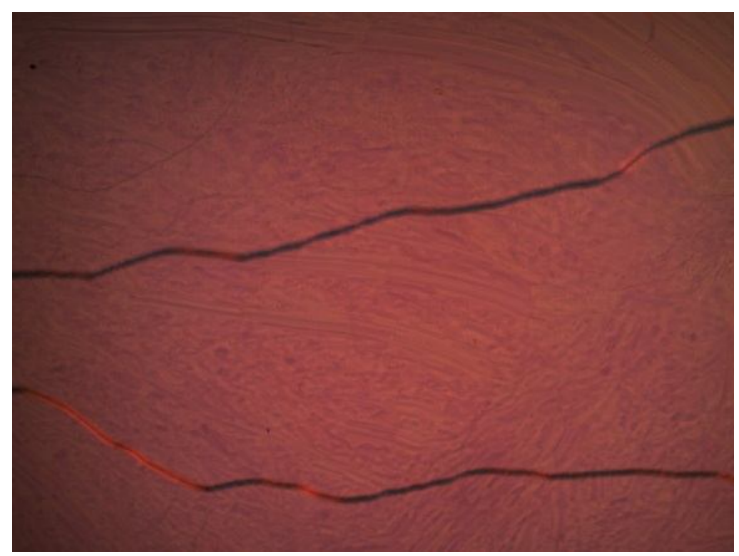

Fig. 2. POM texture observed for the hybrid cell placed in between crossed polarizers in the transmission mode. Thick lines are 'oily streaks' and thin lines resemble a 'fingerprint' texture.

Moreover, we find that more direct evidence can be obtained from the POM observations performed in the reflection mode. In this mode, our sample is illuminated through the microscope objective such that the light reflected from the sample returns back into the objective. In this observation mode, the POM texture is formed by distribution of the director in the vicinity of the upper substrate faced to the objective, which implies that the Grandjean and 'pseudo-fingerprint' 
textures can be observed separately for each of the two surfaces, contrary to the transmission mode where they are superimposed.

In Fig. 3a we show the texture obtained in the reflection mode such that the cell placed on the microscope stage is faced towards the objective by its substrate with planar-anchoring conditions. This texture is nothing but a typical Grandjean texture. Usually the Grandjean texture is observed for the flat cells with planar Ch layers. A clearly visible colour of the selective reflection varies from green to orange. Here the 'psedo-fingerprint' texture is not observed, although it becomes visible whenever one turns the cell by its homeotropic substrate towards the objective (see Fig. 3b). These two textures bring evidence that the cell under study is hybrid, with the planar alignment at one substrate, as visualized by the Grandjean texture, and the homeotropic molecular alignment at the opposite substrate, as visualized by the 'pseudo-fingerprint' texture.

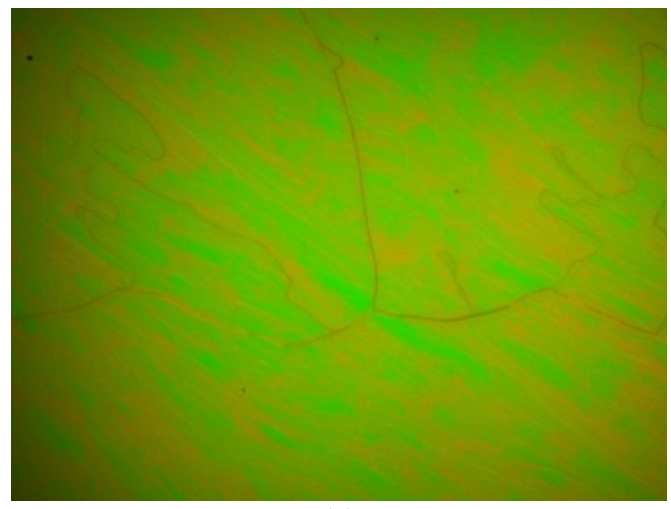

(a)

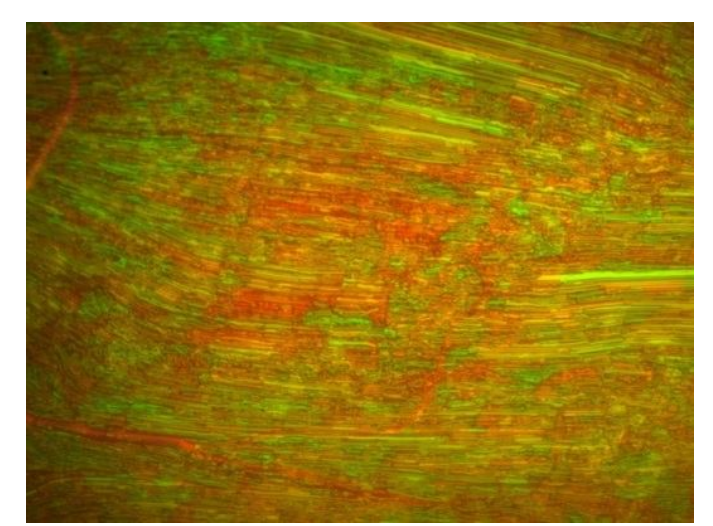

(b)

Fig. 3. POM texture of the hybrid cell observed in the reflection mode, when the light falls upon and reflects from the surface of the substrate with (a) planar and (b) homeotropic boundary conditions for the liquid-crystal molecules.

It should be noticed that for homeotropic cells of long-pitch $\mathrm{Ch}$-s, the periodicity of the 'fingerprint' texture is of the order of half-pitch or a bit larger if the layers are tilted with respect to the substrates. Domains with different periodicities can coexist in the same cell, but their periods usually differ less than twice. The quasi-periodic set of lines seen in the 'pseudo-fingerprint' texture in Fig. 3b has the period much larger $(\sim 4 \mu \mathrm{m})$ than the Ch pitch. We suggest that its origin is analogous to that of the oily-streak and focal conic domain textures observed for thin hybridaligned smectic cells. Therefore the 'pseudo-fingerprint' texture is only apparently similar to the 'fingerprint' texture characteristic of the Ch-s. By its origin it is a typical texture seen for the layered liquid crystals such as smectics. For this reason we call the corresponding texture observed in the hybrid-aligned Ch-s as the 'pseudo-fingerprint' texture.

The Ch pitch can be estimated from the colour of selective reflection, which corresponds roughly to the light wavelength $\lambda_{s} \sim 0.57 \mu \mathrm{m}$. For the $\mathrm{Ch}$ mixture under study, the average refractive index can be taken to be approximately $\bar{n} \sim 1.6$ and, thus, the pitch can be estimated as $P \sim \lambda_{s} / \bar{n} \approx 0.36 \mu \mathrm{m}$. Therefore the pitch and, consequently, the half-pitch $P / 2 \sim 0.18 \mu \mathrm{m}$ appear to be significantly shorter than the typical periodicity $\sim 4 \mu \mathrm{m}$ observed for the lines in Fig. 3b. In its turn, the latter is much smaller than the cell thickness $(d=16.6 \mu \mathrm{m})$.

A set of parallel lines perpendicular to the helix direction, with the periodicity of several pitches, has been documented in Ref. [17] for the short-pitch Ch cell of which substrates are 
treated for providing homeotropic molecular alignment. This texture has been identified as a socalled $\mathrm{Ch}$ focal conic domain texture. Inspection of the quasi-periodic texture shown in Fig. 3b shows that the periodic lines are broken by closely packed circular domains reminiscent of the focal conic domains formed by equidistant curved Ch layers. Then the texture in Fig. $3 b$ can be identified as a set of oily streaks, which split into focal conic domains in some places. The latter is in full analogy with a similar texture observed in hybrid-aligned smectic A cells [18]. Indeed, the short-pitch Ch-s elastically are similar to the smectics and, because of this property, the focal conic domain texture is one of the typical $\mathrm{Ch}$ textures [8]. The diameter of the focal conic domains and the periodicity of the oily streaks in smectics are of the order of the sample thickness, being much larger than the periodicity of smectic layers, which is of the order of the molecular length. In the same vein, the size $D$ of focal conic Ch domains in the hybrid-aligned short-pitch Ch-s [19] and the period of the Ch oily streaks are defined by the thickness of the region in the cell where the layers are curved. It follows from Fig. $3 \mathrm{~b}$ that $D \sim 4 \mu \mathrm{m}$, which is smaller than the sample thickness. This is why we are led to conclude that the Ch layers are strongly curved in the spatial domain $\sim 4 \mu \mathrm{m}$. The POM texture observed in the reflection mode from the substrate coated with the polymer PI2555, together with the fact of detection of lasing in the hybrid-aligned $\mathrm{Ch}$ cell under test, suggest that the remaining space of the cell is filled by parallel Ch layers. We will continue discussion of this issue in Sec. 3.2.

\subsection{Optically pumped emission spectra of the hybrid Ch cell}

Our experiments show that the hybrid $\mathrm{Ch}$ cell reveals laser generation similar to that obtained from the planar $\mathrm{Ch}$ cell, for which the laser peak $(\lambda=605 \mathrm{~nm})$ is recorded at the long-wavelength edge of the Ch PhBG (see Fig. 1a, b). However, instead of a single lasing line, one observes several peaks for the hybrid cell filled with the same $\mathrm{Ch}$ mixture, which are closely grouped around the long-wavelength edge of the $\mathrm{Ch}$ PhBG. The number and the heights of the peaks vary in time.

Fig. 4a displays the separate emission spectra captured at different time moments for the hybrid Ch cell which is pulse-excited with the repetition frequency $f=150 \mathrm{~Hz}$. The same spectra in Fig. $4 \mathrm{~b}$ are superimposed. The fact that the emission peaks shown in Fig. 4 are located at the edge of the Ch PhBG (see Fig. 1a) suggests that these peaks have the same nature as the laser line for the planar Ch cell (see Fig. 1b). The spectral width of the region where the lasing peaks are observed for the hybrid cell is of the order of $10 \mathrm{~nm}(600-610 \mathrm{~nm})$. The laser lines are seen on both sides of the wavelength $\lambda=605 \mathrm{~nm}$ that corresponds to the long-wavelength edge of the PhBG of the planar cell.

The latter would imply that the laser generation takes place in the Ch layers near the substrate coated with the polymer PI2555, where the layers remain roughly parallel to the substrate, though they might be significantly stretched because of their strong distortion in the bulk. It has been shown theoretically and proven experimentally that stable lasing is possible only if the thickness of the planar Ch layers is equal to at least ten Ch pitches [20]. In case of the estimation $P \sim 0.36 \mu \mathrm{m}$ alluded to above, the thickness of the domain with the planar $\mathrm{Ch}$ layers should therefore be at least $4 \mu \mathrm{m}$ (i.e., at least $1 / 4$ of the cell thickness) or larger.

At the end of Sec. 3.1, we have concluded that the thickness of the sample region, where the Ch layers are essentially curved, is of the order of $D \sim 4 \mu \mathrm{m}<<d=16.6 \mu \mathrm{m}$. This agrees with the conclusion that the Ch layers remain parallel to the substrate coated with PI255 within a finite micron distance, which can be estimated as $\Delta d=d-D \sim 12 \mu \mathrm{m}$.

Ukr. J. Phys. Opt. 2017, Volume 18, Issue 3 

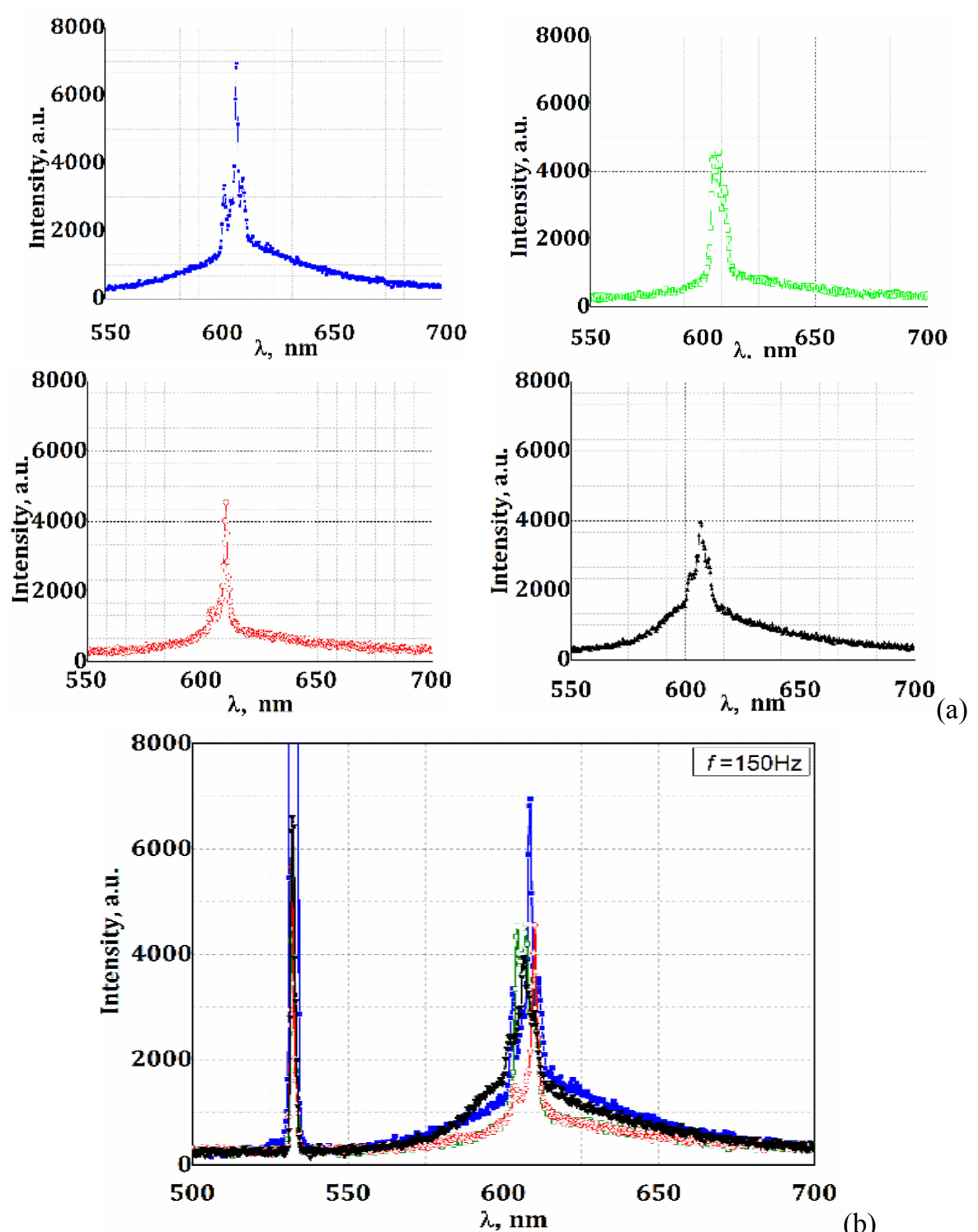

(a)

(b)

Fig. 4. Emission spectra recorded at different time moments for the hybrid Ch cell at the repetition frequency $f=150 \mathrm{~Hz}$ of excitation pulse. Panels (a) and (b) correspond to separate and superimposed spectra, respectively. A peak on the left of $\lambda=532 \mathrm{~nm}$ originates from the excitation laser.

In comparison with the long-pitch Ch-s or their untwisted nematic counterparts, where the orientation of the director varies rather smoothly or even nearly linearly from one substrate to the other, the elasticity and the surface anchoring for the short-pitch $\mathrm{Ch}$-s are more complicated. The director field at the hybrid alignment suffers a bend deformation near the substrate with the homeotropic anchoring and a splay deformation near the substrate with the planar alignment. Energetically, the bend director deformation in Ch-s is comparable to that observed in nematics, whereas because of the orientational $\mathrm{Ch}$ periodicity, the splay deformation costs higher elastic energy [8]. A considerable amount of both theoretical and experimental work has been done to establish organization of $\mathrm{Ch}$ layers for the long-pitch $\mathrm{Chs}$ in the homeotropic and hybrid cells [2125]. Unfortunately, the short-pitch Ch-s remain less studied in this respect. The POM textures and 
the experimental observation of lasing in the hybrid Ch cell reported in the present study suggest that, at the substrate with planar alignment, the layers remain planar for some finite micron distance from the substrate. We estimate it to be approximately $3 / 4$ of the cell thickness.

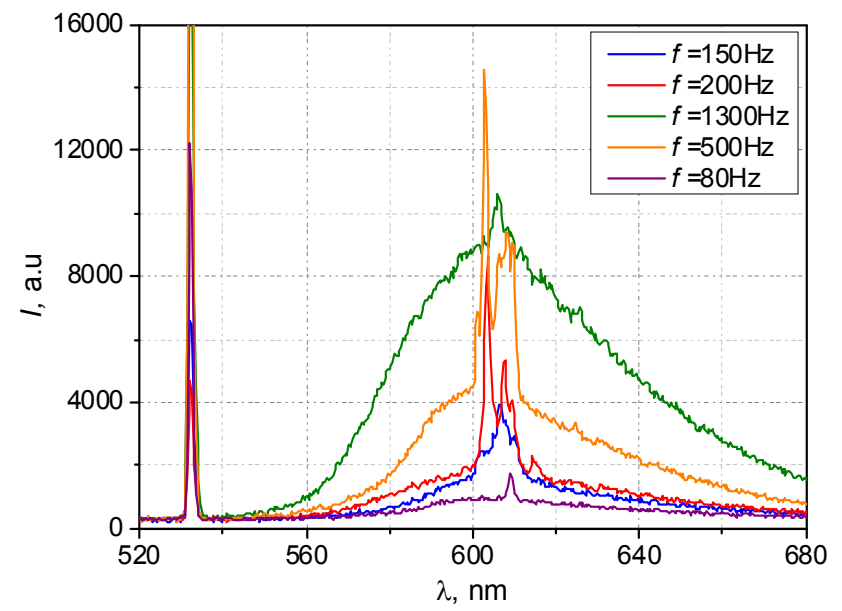

Fig. 5. Emission spectra of the hybrid Ch cells detected under pulse excitation with different repetition frequencies displayed in the legend.

Dilatation of the parallel Ch layers near the substrate coated with the polymer PI2555 should lead to local variations of the $\mathrm{Ch}$ pitch along the substrate, if compared to the equilibrium pitch value. This can be manifested through lasing at different wavelengths around the $\mathrm{Ch}$ PhBG edge.

Redistribution of the emission power among the individual peaks, which is observed in time, indicates that the laser modes corresponding to different lines are highly coupled. As supposed above, multiple lasing lines result, most probably, from varying $\mathrm{Ch}$ pitch. Strong interaction of the laser modes indicates that the spatial zones with different pitches are located close to each other across the spot of the excitation beam $(\sim 500 \mu \mathrm{m}$ in diameter in our experiments). In its turn, the latter implies high density of edge dislocations in the sample. The situation resembles random lasing in strongly inhomogeneous scattering media where different modes are also localized close to each other, such that the separate modes appear to be coupled and strongly interacting with each other [26, 27].

We have also studied dependence of the emission spectra for the hybrid cell on the repetition frequency of the excitation signal (see Fig. 5). If the repetition frequencies are relatively low ( $f=80 \mathrm{~Hz}$ ), there is only one laser line of relatively low intensity (see the purple curve in Fig. 5). The line intensity is low because of low exciting pulse energy peculiar for the low frequencies. The number of the lasing modes and the corresponding peak heights increase with increasing frequency (i.e., at a higher excitation intensity). Dependence of the number of lasing modes on the excitation power indicates that different modes have different excitation-power thresholds. The lowest lasing threshold corresponds to the excitation at $f=80 \mathrm{~Hz}$. It is detected for the line located at $\lambda=605 \mathrm{~nm}$, which is the same as that observed for the planar Ch cell (see Fig. 1b). The intensities of the lasing lines increase with increasing frequency approximately up to $f=500 \mathrm{~Hz}$. When the frequency increases further on, one observes a significant increase in the spontaneous emission, such that its level becomes comparable to the lasing intensity. As a result, the main light power is transferred into non-resonant emission. Then the lasing lines are suppressed and cannot 
be resolved against the background of the spectral band originated from the spontaneous emission. This is clearly seen from the emission spectrum in Fig. 5 that corresponds to the frequency $f=1300 \mathrm{~Hz}$.

\section{Conclusions}

Our experiments demonstrate that the laser generation in dye-doped Ch is possible not only for the cells with high-quality planar orientation of the Ch layers or low-quality planar orientation in the presence of large number of defects. It is also possible in the hybrid cell for which the orientation of $\mathrm{Ch}$ layers changes from planar at one substrate to homeotropic at the opposite substrate. Instead of a single laser line typical for the planar cells, one observes multiple laser lines for the hybrid cell, which are located on the both sides of long-wavelength edge of the PhBG detected for the planar Ch cell. The distribution of the emitted energy between these lines varies in time. Increasing repetition frequency of the excitation signal leads to increased intensity and number of the laser lines, as well as to increased intensity of non-resonant radiation that consumes the energy. This inhibits amplification of the laser modes at high enough repetition frequencies of the excitation signal. At the frequencies higher than $1500 \mathrm{~Hz}$, the intensities of the laser lines become comparable to the level of background non-resonant modes and, as a result, the laser lines cannot be resolved in the emission spectrum.

The other problem solved in this study is identification of the hybrid alignment in the shortpitch $\mathrm{Ch}$ cell. We demonstrate that the short-pitch hybrid-aligned $\mathrm{Ch}$ cell displays distinctive textures in the reflection modes. Namely, we observe the Grandjean texture for the substrate under the planar-alignment conditions and the 'pseudo-fingerprint' texture characteristic for the homeotropic-anchoring conditions. The latter is an analogue of the oily streaks and the focal conic domain textures observed for the hybrid-aligned smectics. We prove that curved and planar $\mathrm{Ch}$ layers coexist in our cell, such that the latter region occupies about $3 / 4$ of the cell thickness. The presence of such a thick domain with planar Ch layers is supported by the fact that the lasing is detected at the edge of the $\mathrm{Ch} \mathrm{PhBG}$.

\section{References}

1. Kopp V I, Fan B, Vithana H K M and Genack A Z, 1998. Low-threshold lasing at the edge of a photonic stop band in cholesteric liquid crystals. Opt. Lett. 23: 1707-1709.

2. Coles H and Morris S, 2010. Liquid-crystal lasers. Nature Photonics. 4: 676-685.

3. Dudok T H and Nastishin Yu A, 2014. Optically pumped mirrorless lasing. A review. Part II. Lasing in photonic crystals and microcavities. Ukr. J. Phys. Opt. 15: 47-67.

4. Chapran M, Angioni E, John N, Breig F B, Cherpak V, Stakhira P, Tuttle T, Volyniuk D, Grazulevicius J V, Nastishin Yu A, Lavrentovich O D and Skabara P J, 2017. An ambipolar BODIPY derivative for a white exciplex OLED and cholesteric liquid crystal laser towards multi-functional devices. Appl. Mater. \& Interfaces. 9: 4750-4757.

5. Morris S M, Ford A D, Gillespie C, Pivnenko M N, Hadeler O and Coles H J, 2006. The emission characteristics of liquid-crystal lasers. J. Soc. Inf. Disp. 14: 565-573.

6. Dudok T H, Savaryn V I, Krupych O M, Fechan A V, Lychkovskyy E, Cherpak V V, Pansu B and Nastishin Yu A, 2015. Lasing in imperfectly aligned cholesterics. Appl. Opt. 54: 96449653.

7. Dudok T H, Savaryn V I, Meyer C, Cherpak V V, Fechan A V, Lychkovskyy E I, Pansu B and Nastishin Yu A, 2016. Lasing cholesteric capsules. Ukr. J. Phys. Opt. 17: 169-175. 
8. Kleman M, Lavrentovich $\mathrm{O} \mathrm{D}$ and Nastishin $\mathrm{Yu}$ A. Dislocation and disclination in mesomorphic phases. In: Dislocations in solids, vol. 12. Ed. by F R N Nabarro and J P Hirth. Elsevier (2004). pp. 147-271.

9. Nastishin Yu A, Polak R D, Shiyanovskii S V, Bodnar V H and Lavrentovich O D, 1999. Nematic polar anchoring strength measured by electric field techniques. J. Appl. Phys. 86: 4199-4213.

10. Lavrentovich O D, 2003. Fluorescence confocal polarizing microscopy: three-dimensional imaging of the director. Pramana J. Phys. 61: 373-384.

11. Nastyshyn S Yu, Bolesta I M, Lychkovskyy E, Vankevych P I, Yakovlev M Yu, Pansu B and Nastishin Yu A, 2017. Ray tracing matrix approach for refractive index mismatch aberrations in confocal microscopy. Appl. Opt. 56: 2467-2475.

12. Shribak $M$ and Oldenbourg R, 2003. Techniques for fast and sensitive measurements of two-dimensional birefringence distributions. Appl. Opt. 42: 3009-3017.

13. Stetsyshyn Yu, Raczkowska J, Budkowski A, Awsiuk K, Kostruba A, Nastyshyn S Yu, Harhay Kh, Lychkovskyy E, Ohar H and Nastishin Yu A, 2016. Cholesterol-based grafted polymer brushes as alignment coating with temperature-tuned anchoring for nematic liquid crystals. Langmuir. 32: 11029-11038.

14. Scheffer T J and Nehring J, 1977. Accurate determination of liquid-crystal tilt bias angles J. Appl. Phys. 48: 1783-1792.

15. Andrienko D, Kurioz Y, Reznikov Y, Rosenblatt C, Petschek R, Lavrentovich O and Subacius D, 1998. J. Appl. Phys. 83: 50-55.

16. Ziherl P, Subacius D, Strigazzi A, Pergamenshchik V M, Alexe-Ionescu A L, Lavrentovich O $\mathrm{D}$ and Zumer S, 1998. Magnetic field controlled optical phase retardationin a hybrid nematic cell. Liq. Cryst. 24: 607-612.

17. Salter P S, Elston S J, Raynes P and Parry-Jones L A. 2009. Alignment of the uniform lying helix structure in cholesteric liquid crystals. Jap. J. Appl. Phys. 48: 101302-5.

18. Gryn I, Lacaze E, Bartolino R and Zappone B, 2014. Controlling the self-assembly of periodic defect patterns in smectic liquid crystal films with electric fields. Adv. Funct. Mater. 25: 142149.

19. Nastishin Yu A, Kleman M and Dovgyi O B, 2002. Textural and conoscopic studies of chiral liquid crystals possessing cholesteric - smectic A or cholesteric - TGBA - smectic A phase transitions. Ukr. J. Phys. Opt. 3: 1-11.

20. Dowling J P, Scalora M, Bloemer M J and Bowden Ch M, 1994. The photonic band edge laser: A new approach to gain enhancement. J. Appl. Phys. 75: 1896-1899.

21. Dozov I and Penchev I. 1986. Structure of a hybrid aligned cholesteric liquid crystal cell. J. de Physique. 47: 373-377.

22. Lewis M R and Wiltshire M C K, 1987. Hybrid aligned cholesteric: A novel liquid-crystal alignment. Appl. Phys. Lett. 51: 1197-1199.

23. Lin Ch-H, Chiang R-H, Liu Sh-H, Kuo Ch-T and Huang Ch-Y, 2012. Rotatable diffractive gratings based on hybrid-aligned cholesteric liquid crystals. Opt. Express. 20: 26837-26844.

24. Nose T, Miyanishi T, Aizawa Y, Ito R and Honma M. 2010. Rotational behavior of stripe domains appearing in hybrid aligned chiral nematic liquid crystal cells. Jap. J. Appl. Phys. 49: 051701-5.

25. Shiyanovskii S V and Lavrentovich O D, 2003. 3D simulations of nematic and cholesteric liquid crystals in complex geometries. SID Intnl. Digest Tech. Papers XXXIV. 34: 664-667.

Ukr. J. Phys. Opt. 2017, Volume 18, Issue 3 
26. Karen L van der Molen, R Willem Tjerkstra, Allard P Mosk, and Ad Lagendijk, 2007. Spatial extent of random laser modes. Phys. Rev. Lett. 98: 143901-4.

27. Nastishin Yu A and Dudok T H, 2013. Optically pumped mirrorless lasing. A review. Part I. Random lasing. Ukr. J. Phys. Opt. 14: 146-170.

Nastishin Yu.A., Dudok T.H., Hrabchak V.I., Lychkovskyy E., Yakovlev M.Yu., Vankevych P.I., Meyer C. and Pansu B. 2017. Lasing in a hybrid-aligned cholesteric. Ukr.J.Phys.Opt. 18: 121-130.

Анотація. Зареєстровано лазерну генерацію на довгохвильовому краю фотонної щілини холестерика при оптичному нагнітанні легованої барвником холестеричної комірки, яка задає гібридну орієнтацію так, щзо молекули холестерика паралельні до підкладки поблизу однієї з підкладок, але перпендикулярні поблизу іншої підкладки. Гібридну орієнтацію $в$ коміриі підтверджено спостереженнями в поляризаційному оптичному мікроскопі $в$ режимах пропускання та відбивання. 